\title{
MEMS Incandescent Light Source
}

\author{
Margaret Tuma ${ }^{\mathrm{a}}$, Kevin King ${ }^{\mathrm{b}}$, Lynn Kim ${ }^{\mathrm{b}}$, Richard Hansler ${ }^{\mathrm{c}}$, Eric Jones ${ }^{\mathrm{b}}$, Thomas George ${ }^{\mathrm{b}}$ \\ ${ }^{a}$ NASA Glenn Research Center \\ 21000 Brookpark Road, MS 77-1 \\ Cleveland, $\mathrm{OH} 44135$ \\ ${ }^{\mathrm{b}}$ Jet Propulsion Laboratory, MS 302-231 \\ 4800 Oak Grove Dr. \\ Pasadena, CA 91109 \\ ${ }^{c}$ Lighting Innovations Institute \\ 20700 North Park Blvd. \\ University Heights, OH 44118
}

\begin{abstract}
A MEMS-based, low-power, incandescent light source is being developed. This light source is fabricated using three bonded chips. The bottom chip consists of a reflector on Silicon, the middle chip contains a Tungsten filament bonded to Silicon and the top layer is a transparent window. A $25-\mu \mathrm{m}$-thick spiral filament is fabricated in Tungsten using lithography and wetetching. A proof-of-concept device has been fabricated and tested in a vacuum chamber. Results indicate that the filament is electrically heated to approximately $2650 \mathrm{~K}$. The power required to drive the proof-of-concept spiral filament to incandescence is $1.25 \mathrm{~W}$. The emitted optical power is expected to be approximately $1.0 \mathrm{~W}$ with the spectral peak at $1.1 \mu \mathrm{m}$. The micromachining techniques used to fabricate this light source can be applied to other MEMS devices.
\end{abstract}

Keywords: MEMS light source, broadband source, incandescent source

\section{INTRODUCTION}

The initial motivation for this research was based on 1997 flight test results on a Fabry-Perot fiber-optic temperature sensor system $^{1}$ used to detect exhaust gas temperature (EGT). While the feasibility of the sensor system was proven, the commercial light source optically powering the device was identified as a critical component requiring improvement. Reasons for this include: the source required a long stabilization time ( 1 hour $)$, emitted an enormous amount of heat, and required approximately 6.5 Watts of input electrical power. Thus, it was determined that a new light source needed to be developed to enable broadband optical sensors to be utilized in aerospace applications. A NASA Glenn (GRC), Jet Propulsion Laboratory (JPL), and Lighting Innovations Institute (LII) team was subsequently formed. A seed program was initiated to develop miniature, long-lived, broadband light sources for aerospace applications. The team began by conducting a survey of state-ofthe-art light source technology. The team determined that an incandescent filament light source was the best choice for a miniature, low-power emitter with a broad $(400-1000 \mathrm{~nm})$ spectral range.

Semiconductor chip-based light sources, such as lasers and light emitting diodes, have a relatively narrow range of emission wavelengths in comparison to incandescent sources. Incandescent light sources emit broadband radiation from the visible to the infrared (IR) wavelengths; the intensity at each wavelength is determined by the filament temperature and the materials chosen for the filament and the lamp window. However, present commercial incandescent light sources are large in size and inefficient, requiring several watts of electrical power in order to obtain the desired optical power and emit a large percentage of the input power as heat that must be dissipated.

MEMS-based micro-lamps were developed in the early 1990's, initially using poly-Silicon filaments, ${ }^{2}$ and later thin-film $(<3$ $\mu \mathrm{m})$ Tungsten filaments. ${ }^{3}$ The primary applications for these sources were infrared and optical spectroscopy. However, the operating temperature of the poly-Silicon sources was limited to $1400 \mathrm{~K}$, while the thin-film Tungsten sources had limited lifetimes ( $<1$ hour) due to evaporative material loss.

This is a preprint or reprint of a paper intended for presentation at a conference. Because changes may be made before formal publication, this is made available with the understanding that it will not be cited or reproduced without the permission of the author. 
The miniature light source described in this paper requires $1 / 5$ the electrical input power of some commercial light sources, while providing similar output light power that is easily coupled to an optical fiber. Furthermore, it is small, rugged, and lightweight. Advancements in micro-fabrication technology were used to reduce the size, weight, power consumption and potential cost of miniature broadband light-sources, parameters critical to future aerospace applications. This chip-based light source has the potential for monolithic fabrication with on-chip drive electronics. Other uses for these light sources are in systems for vehicle navigation, remote sensing applications such as monitoring bridges for stress, calibration sources for spectrometers, light sources for space sensors, display lighting, addressable arrays, and industrial plant monitoring.

The basic principles underlying the design, fabrication and operation are being investigated. The proof-of-concept for the micro-fabricated light source has been demonstrated. From this, a prototype design has been completed and fabrication is underway. A summary of the results from these investigations along with the directions for future work will be presented.

\section{PROOF-OF-CONCEPT DEVICE AND RESULTS}

MEMS techniques offer the advantage of fabricating ultra-low mass, low power, monolithic light-sources that can be easily integrated with electronics and fiber optics to produce a compact device. In order to obtain sufficient emissive power at the shorter, visible wavelengths, it was determined that the filament had to operate at temperatures near $2500 \mathrm{~K}$, making Tungsten (W) the material of choice for the filament. As for satisfying the long lifetime requirement $(>10,000$ hours of operation), it was determined that the filament would have to be at least $10 \mu \mathrm{m}$ thick in order to compensate for evaporative losses. MEMS techniques allow the fabrication of planar filaments, which provide higher optical throughput to fiber optics due to (1) the close proximity of the window and the coupling optics to the planar filament and (2) the circular image of the filament matching the circular shape of the fiber optics. This stage of development of the project culminated with the successful fabrication and demonstration of a proof-of-principle lithographically patterned planar Tungsten filament at 2650 K (Figure 1). ${ }^{4,5}$

To calculate the total expected output optical power, the filament surface area must be determined, as shown in Equation (1), where the filament coil has a radius of $0.07 \mathrm{~cm}$.

$$
\text { Surface Area }=\pi * \mathrm{r}^{2}=0.015 \mathrm{~cm}^{2}
$$

Because the planar filament has two sides, there is a multiplication factor of two for the surface area, but because the fill factor, defined as the ratio of filament material to the total area, is $1 / 2$, the two terms cancel. This indicates that the radiant power should simply be the product of the total radiation intensity for Tungsten, 110 Watts/square $\mathrm{cm}$ at $2800 \mathrm{~K},{ }^{6}$ and the filament area as given in Equation (2).

$$
110\left(\text { Watts } / \mathrm{cm}^{2}\right) * 0.015\left(\mathrm{~cm}^{2}\right)=1.69 \mathrm{~W}
$$

Since the goal is to develop a long-life filament, a $2500 \mathrm{~K}$ operating temperature is more realistic. If the filament is heated to $2500 \mathrm{~K}$, the total radiation intensity drops in value from 110 to $66.1,{ }^{6}$ which means the filament will radiate $0.992 \mathrm{~W}$. For a fill factor of $25 \%$ instead of $50 \%$, the radiant power would further drop to $0.496 \mathrm{~W}$. Thus, the higher the filament temperature and fill factor, the greater the radiant power.

Optical output of the proof-of-concept light source was collected through a vacuum chamber window. Measurements were taken using an Analytical Spectral Devices (ASD) Fields Spectrometer, which can measure from $350 \mathrm{~nm}$ to $2500 \mathrm{~nm}$. The data was acquired continuously and can be saved on command. The spectrometer was optimized using a standard $1000 \mathrm{~W}$ lamp and a spectralon panel that is $\sim 99 \%$ reflective. A 38 -fiber cable (some fibers for visible, some for IR) was used to transfer the light from the source to the spectrometer with an 8-degree field-of-view. The light source was driven at $170 \mathrm{~mA}$ at $7.356 \mathrm{~V}$, providing an input power of $1.25 \mathrm{~W}$. A plot of light intensity vs. wavelength is shown in Figure 2. Two discontinuities in the graph are obvious, one near $950 \mathrm{~nm}$ and the other near $1800 \mathrm{~nm}$, which are attributed to the changing of detectors. A blackbody approximation was fit to the experimental data, which gave a blackbody temperature of $2650 \mathrm{~K}$.

Although the test was promising, there were several problems with the approach. Since conventional deposition techniques such as sputtering and evaporation cannot produce thick, stress-free films in short times, a hybrid approach was adopted for the proof-of-concept demonstration. A $25-\mu \mathrm{m}$-thick Tungsten sheet was lithographically patterned and etched using wet chemical techniques to form a planar spiral. Due to the isotropic nature of the wet etchant, the fill-factor is approximately $50 \%$. This limitation of the wet-etch technique clearly requires improvement in order to produce a densely packed filament 


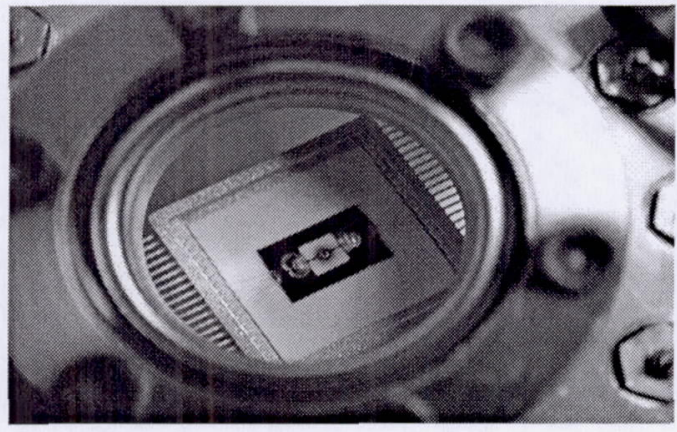

(a)

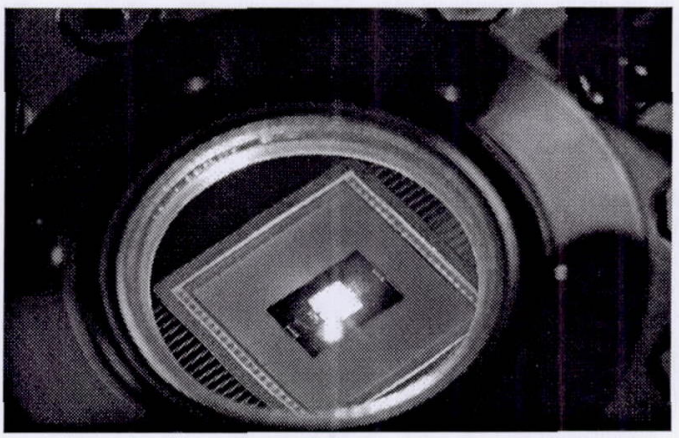

(b)

Figure 1. (a) Proof-of-concept light source in vacuum chamber and (b) Proof-of-concept demonstration of MEMS light source conducted at $2650 \mathrm{~K}$ in a vacuum chamber.

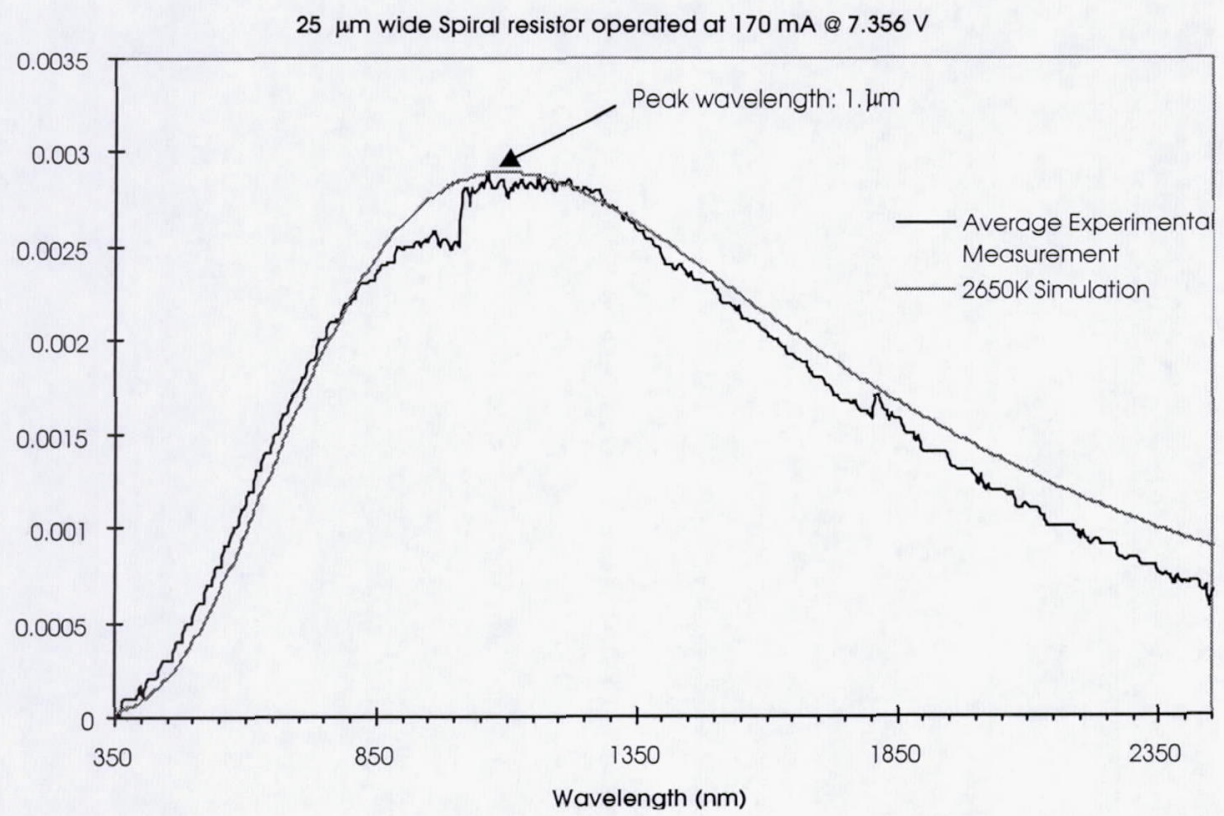

Figure 2. Emission spectrum of the proof-of-concept Tungsten filament source. (The jagged line denotes experimental data where the discrete steps are due to measurement artifacts. The smooth line is the simulated $2650 \mathrm{~K}$ blackbody curve.)

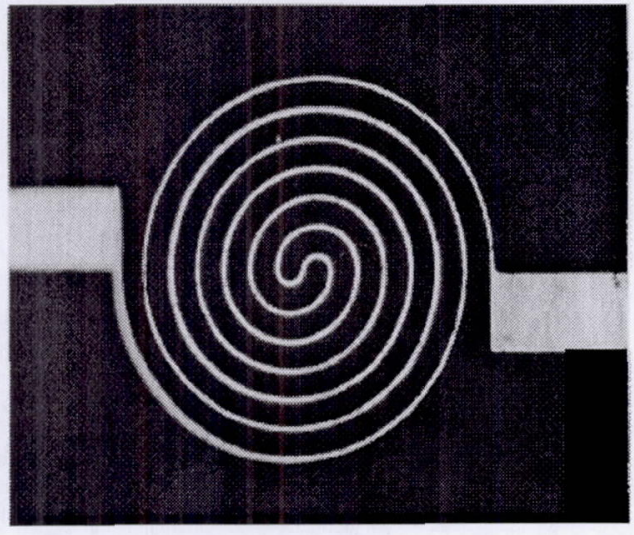

(a)

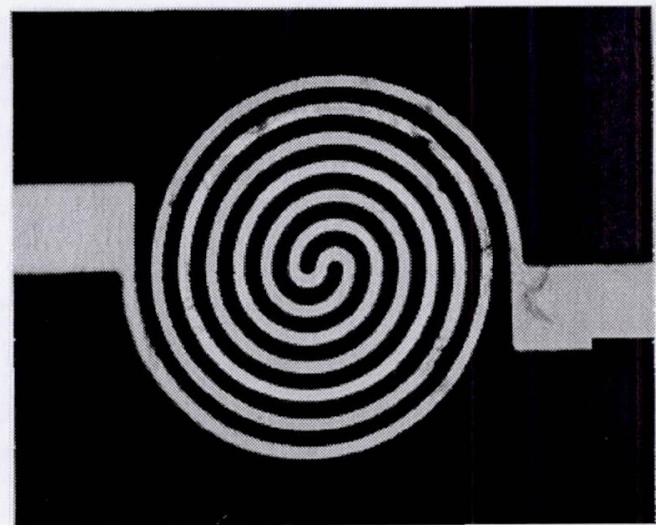

(b)

Figure 3. Photographs at 50X magnification of Tungsten spiral filaments wet-etched from a 25 - $\mu$ m-thick Tungsten sheet. (a) $25-\mu \mathrm{m}$-wide spiral with $75-\mu \mathrm{m}$ spacing between adjacent spirals (25\% fill-factor) and (b) $50-\mu \mathrm{m}$ wide spirals with $50-\mu \mathrm{m}$ spacing (50\% fill factor). 
with a higher fill-factor and, hence, a smaller, more intense and uniformly distributed light source. Figure 3 shows two spirals with different fill-factors, where each spiral is $1400 \mu \mathrm{m}$ in diameter. Ideally, the distance between adjacent Tungsten coils in a spiral should be on the order of $5 \mu \mathrm{m}$ ( $90 \%$ fill factor), where shorting and/or arcing are the limiting factors. This will enable the maximum amount of optical energy to be coupled into a fiber. The current hybrid approach of attaching etched tungsten filaments onto prefabricated Silicon chips is not ideal from both a reliability and mass production standpoint. Laser-based fabrication processes will likely be the most promising for producing a reliable, fully integrated, long-life, planar tungsten filament. The ultimate goal of the research is to develop a long-lived $(\sim 10,000 \mathrm{hr})$ stable light-source. Additionally, the hybrid approach had attendant problems of high contact resistance, which varied with thermal expansion related relative motion of the filament between the clamping contacts. Thus, of all possible fabrication techniques, laser-based processing offers the best chance to obtain high fill-factor, monolithic, planar Tungsten filaments.

\section{PROTOTYPE DEVICE}

Based upon the results of the proof-of-concept device, a prototype device geometry was conceived. The prototype consists of a 3-wafer stack: (A) a top transmission window, (B) a middle filament mount, and (C) a bottom reflector. An exploded 3-D view of the 3-wafer stack is shown in Figure 4 and a 2-D cross-section of the 3-wafer stack (side view) is shown in Figure 5.

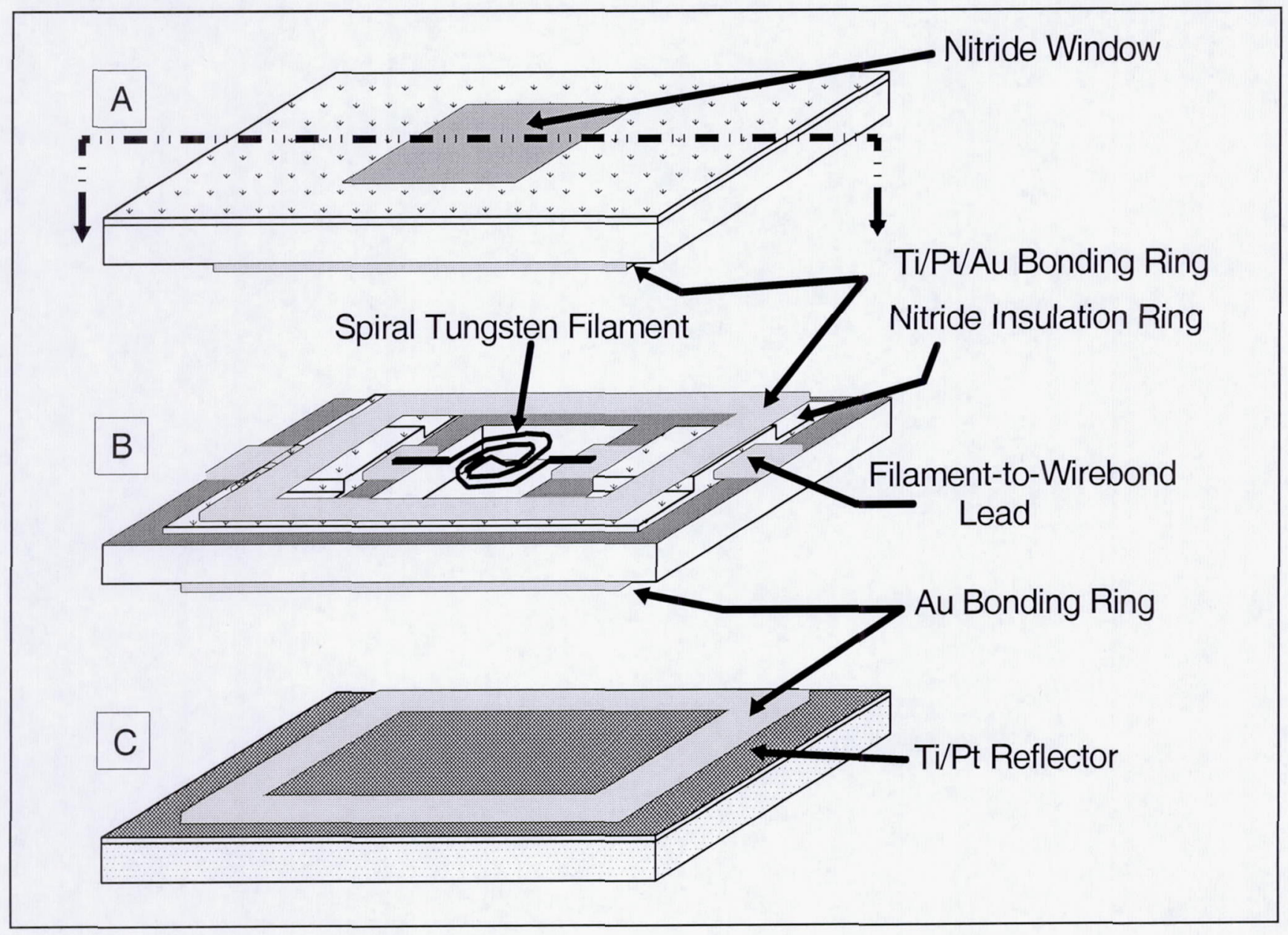

Figure 4. Exploded view of 3-wafer stack: (A) Transmission Window, (B) Filament Mount, and (C) Reflector 


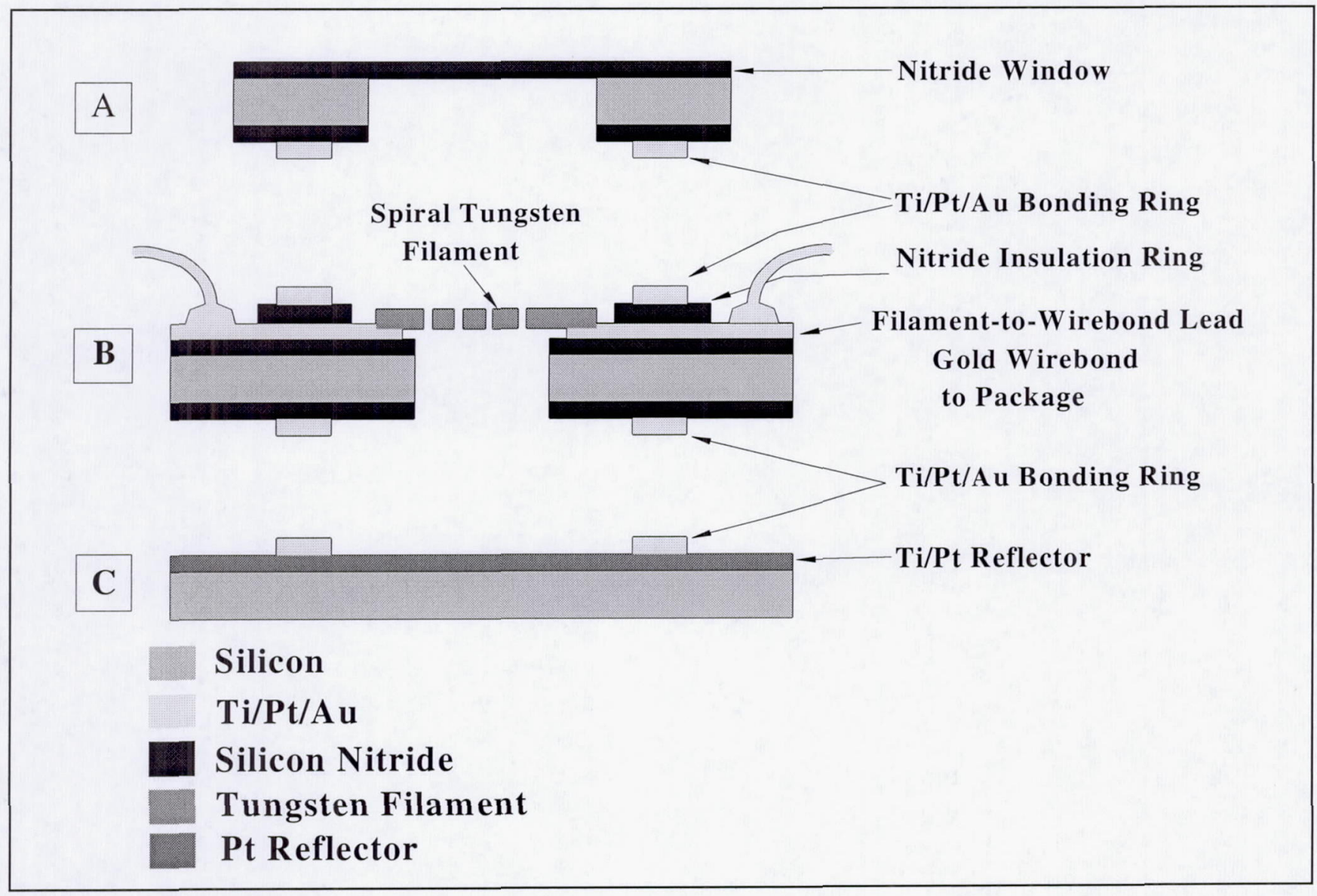

Figure 5. Cross-section of 3-wafer stack along dotted line shown in Figure 4.

(A) Transmission window, (B) Filament mount, and (C) Reflector.

The Silicon substrates used to construct the 3-wafer stack are $400 \mu \mathrm{m}$ in thickness. The physical dimensions of wafer B and $\mathrm{C}$ are $5.40 \mathrm{~mm} \times 8.00 \mathrm{~mm}$. Wafer A is $5.00 \mathrm{~mm} \times 7.20 \mathrm{~mm}$ to provide sufficient area on Wafer B for wire bonds and to prevent an electrical short between the two wafers. The outer dimensions of the 3 -wafer stack are $5.4 \mathrm{~mm} \times 8.0 \mathrm{~mm} \times 1.2$ $\mathrm{mm}$. To test the prototype design, a packaged prototype has been fabricated, without the filament. The next step is to package a prototype with a filament enclosed.

\section{A. Transmission Window Fabrication}

The top substrate functions as a transmission window and is bonded to the middle substrate by Au/Au thermal compression bonding. Initially, a $1000 \AA$ PECVD low-stress Silicon Nitride film is deposited onto $400-\mu \mathrm{m}$-thick bare Silicon. Silicon Nitride was chosen because it is optically transparent between $0.5 \mu \mathrm{m}$ and $8 \mu \mathrm{m} .^{7}$ A Ti/Pt/Au bonding ring $(200 / 200 / 2000 \AA)$ is then evaporated onto the unpolished side using a lift-off process. Finally, the bottom Nitride and the bulk Silicon are etched to reveal the top Nitride window. This opening is wider than the filament length (spiral diameter plus leads on both ends), allowing the $25 \mu$ m-thick-filament mounted to the middle substrate (shown in B) to fit inside. A 500 - $\mu \mathrm{m}$ pyrex coverslip is being considered as an alternate material for the transmission window. Both pyrex and Nitride are transparent in the wavelength of interest $(500-900 \mathrm{~nm})$ as seen in the transmission measurements (Figures 6 and 7). These measurements were taken using an Acton "SpectraPro 275" spectrometer and an incandescent source. Intensity measurements were made in two runs, one with the test window in front of the slit and another run without it. The ratio of the intensity measured during the two runs was calculated and plotted as the fraction of transmitted light. The transmission data through the Silicon Nitride window is oscillatory due to interference in the thin membrane. This Silicon Nitride membrane, while integrated into the top substrate, is fragile and may yield under the large pressure gradient created after vacuum packaging. A pyrex coverslip is more mechanically robust than a Silicon Nitride membrane, but requires more fabrication steps and adds thickness to the transmission window and the overall final device. Fabrication of a pyrex window device can be accomplished by eliminating the Nitride deposition and bonding pyrex to Silicon anodically, or by depositing Ti/Pt/Au bonding rings on the top of substrate A (without the top Nitride layer) and on the bottom of the pyrex for thermal compression bonding. The prototype 
device design shown in Figures 4 and 5 has integrated Nitride membranes. Fabrication of pyrex-windowed devices is in progress.

\section{B. Filament Mount Fabrication}

The middle substrate provides a mount for the Tungsten filament, an electrical conduit through the hermetically sealed wafer stack, and a pad for wire bonding to the external packaging. First, $1000 \AA$ of PECVD Silicon Nitride is deposited on a bare Silicon wafer. Then, two leads are evaporated in the radial direction to electrically connect the filament with the wire bond pads. Next, $1 \mu \mathrm{m}$ of PECVD Silicon Nitride is deposited to insulate the leads. It is patterned in a ring and etched in a Fluorine-based reactive ion etch (RIE) which has been optimized to avoid etching the underlying lead appreciably. A Ti/Pt/Au ring (200/200/2000 $\AA$ ) is evaporated onto the Nitride insulation ring using a lift-off process. This ring will align to the ring on the substrate above (substrate A) during thermal compression bonding. A Ti/Pt/Au ring (200/200/2000 $\AA$ ) is then deposited on the unpolished side using a lift-off process and functions as a bonding ring to the substrate below (substrate $\mathrm{C}$ ). Finally, deep reactive-ion etching is used to etch a 3.9 × $2.9 \mathrm{~mm}$ hole through the entire Silicon wafer.

\section{Filament Fabrication}

Tungsten filaments are fabricated from a $25-\mu \mathrm{m}$-thick Tungsten sheet. Photoresist is patterned on both sides of the sheet with the filament geometry, and the sheet is chemically etched from both sides in a $\mathrm{K}_{3} \mathrm{Fe}(\mathrm{CN})_{6}$ etch. $^{8}$ This was sufficient to etch the entire thickness of the sheet. Etching from both sides reduces lateral etching from $25 \mu \mathrm{m}$ to $12.5 \mu \mathrm{m}$ and thereby improves the final device fill factor. Other methods such as laser etching are being explored to further improve filament fill factor.

\section{Reflector Fabrication}

The bottom reflector provides a reflective surface to improve transmission through the transmission window above by reflecting a portion of the bottom filament radiation to the window. It is fabricated by evaporating a Ti/Pt (200/1000 $)$ reflective film onto a bare Silicon wafer. A Ti/Pt/Au ring (200/200/2000 $\AA$ ) is then evaporated onto the reflective layer for thermal compression bonding to the middle substrate. In future devices, Silver ( $\mathrm{Ag}$ ) will be used as the reflective surface as it is more highly reflective than Pt over the visible-IR wavelength region.

\section{Assembly and Packaging}

The substrates are individually diced after the fabrication steps described above have been performed. The filament is attached to the middle substrate by two Gold ribbons. Other methods of filament attachment such as thermal compression bonding or Au eutectic bonding are presently being explored. Finally, the entire stack is bonded in a thermal compression bonder. To ensure hermetic sealing, the bonding step is performed in an evacuated chamber $\left(<10^{-6} \mathrm{Torr}\right)$, or a backfilled chamber of either pure or halogen-doped $\mathrm{N}_{2}$. To avoid damaging the fragile Silicon Nitride window, a flat pyrex piece, approximately the same size as substrate A, is placed on substrate A and a graphite piece is placed on top of the pyrex to protect the device against surface roughness in the compression bonder piston. Once the 3-wafer stack with filament is assembled, wire bonds are used to connect the stack to a more robust ceramic package.

The transmission through the upper pyrex window and Silicon Nitride window were measured experimentally as described in section A above. Results from the measurements are shown in Figures 6 and 7. A different material, such as sapphire, would be chosen for the window if one were also interested in using the infrared (IR) portion of the wavelength spectrum. Thus, by changing the optical window, one can tailor the output optical spectrum to a particular application.

\section{FUTURE WORK}

\section{Device Fabrication and Packaging}

Packaging studies with filaments processed using the wet-etch technique will begin shortly. The two critical packaging issues are filament attachment and device seal with different ambient gases. Ideally, resistive heating should only occur at the filament itself. However, results from the proof-of-concept device indicate that the contact resistance of the filament and bond pad junctions can be high during filament operation due to thermal expansion of the filament. The proof-of-concept hybrid-filament was attached using a rather crude, Gold strap bonding technique using a ribbon bonder. Two different approaches have been taken to lower contact resistance: spot welding and eutectic bonding. The second issue, device seal, 
directly impacts the lifetime of the filament. The ambient inside the sealed device must be free of oxygen and water vapor, which can quickly oxidize the hot filament. A sealing chamber will be built that can be pressurized with the appropriate gas. The ambient environments currently under consideration are vacuum, nitrogen, and halogen-doped nitrogen. Halogen doping addresses the issue of Tungsten condensation onto the window during operation, which will decrease transmission over time. The halogen will set in motion a chemical reaction cycle that will remove the Tungsten off the window and redeposit it on the filament. $^{9}$

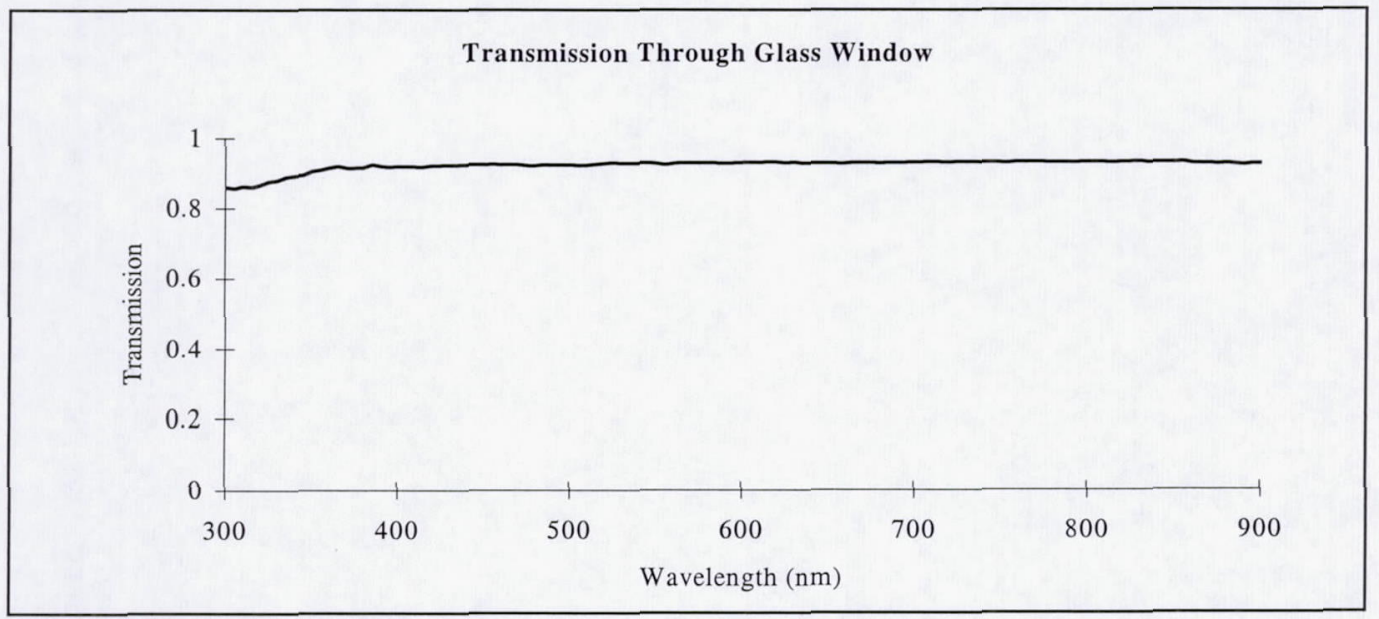

Figure 6. Optical transmission through pyrex window chip vs. wavelength.

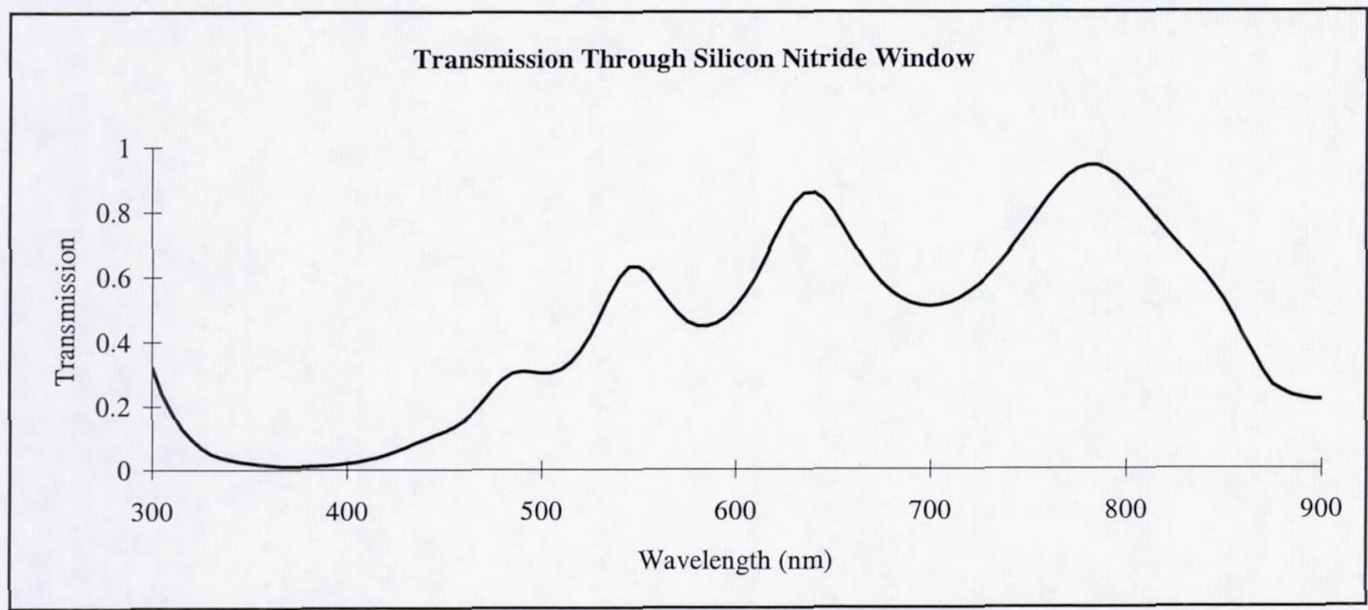

Figure 7. Optical transmission through Silicon Nitride window vs. wavelength.

\section{Fiber optic coupling and testing}

One of the goals of this project is to efficiently couple the output of the device into an optical fiber. Computer modeling of the device will be performed to determine the optimum coupling technique. Results will allow for the addition of fiber coupling into the device design. When a Tungsten filament is heated for the first time, there is a tendency for it to distort slightly due to grain growth and the forces from thermal expansion. This may cause part of the planar filament to move in or out of plane. If the filament is coupled to the fiber by a normal two-ball lens system, a small change in the position of the source will be imaged as a small change in the image position. Because the image of the filament is considerably larger than the core of the fiber, this movement will have negligible effect on the amount of light entering the fiber. Thus, we expect stable coupling efficiency of the output power of this device. If the structure is set before the optics are assembled, any distortion in the filament may be corrected by the placement of the optics. 
A lens structure for the packaged device to maximize the coupling efficiency into a standard optical fiber will be designed. This lens will likely be fabricated into the upper window. The fiber optics and coupling optics will be integrated with the miniature calibration source as per the design developed in the previous phase. The results of modeling using LightTools ${ }^{\mathrm{TM}}$ software will be used to determine the optimum fiber coupling method. Two coupling choices will be considered: (1) direct coupling, using ball lenses, (2) coupling using a graded-index (GRIN) lens integrated into the optical transmission window, and (3) direct coupling without lenses. An optical feedback scheme will be explored as well to insure that the coupled output is stable. Initial alignment and coupling tests will be performed using spectrometers and custom parts. Once the preliminary coupling and alignment have been completed, we will begin long-term characterization of the coupling efficiencies and lifetime and reliability tests of the light source.

\section{Thermal}

More extensive thermal studies will be performed to better determine the required spacing between the filament and window and between the filament and reflector. Results will also provide insight as to the required packaging scheme. Particular attention will be paid to the expected temperature of the upper window and lower reflector chip as well as contacts. Because of the small geometry of the device and the high temperature of the filament, these studies are crucial to ensure a long-lived, durable device.

\section{SUMMARY}

A proof-of-concept device has been fabricated using MEMS techniques and tested. Initial testing has demonstrated that the wet-etched Tungsten filament can be operated up to $2650 \mathrm{~K}$. Results were used to design a prototype light source, consisting of a 3-wafer stack with a Tungsten filament. A prototype package has been fabricated (without a filament). Significant progress has been made in fabrication of both the Tungsten filament and MEMS packaging. Integration of the filament into a vacuum-sealed 3-wafer stack will be performed in the near future. Other future work entails reliability and lifetime tests on the device with the initial goal of a 1000-hour-lifetime.

\section{REFERENCES}

1. M.L. Tuma, T. Sawatari and Y. Lin, "Results of Flight Tests on a Fiber-Optic Temperature Sensor," $45^{\text {th }}$ International Instrumentation Symposium, Albuquerque, NM, 1999.

2. C. H. Mastrangelo, J. H-J. Yeh and R. S. Muller, "Electrical and Optical Characteristics of Vacuum Sealed Microlamps," IEEE Trans. On Electron Devices, 39, 1363, 1992.

3. K. R. Williams and R. S. Muller, "Micromachined Hot-Filament Ionization Pressure Sensor and Magnetometer," Int. Conf. on Solid-State Sensors and Actuators, Transducers, 1997.

4. T. George, E. W. Jones, M. L. Tuma, "Silicon Micromachined Broad Band Light Source," NASA New Technology Report, NPO-20655, 1999.

5. T. George, M.L. Tuma, E.W. Jones, R. Hansler, and M. Eastwood, patent "Silicon Micromachined Broadband Light Source" filed July 10, 2000.

6. W.E. Forsythe, E. Adams, J. Opt. Soc. Am., 35, pp. 108-113, 1945.

7. T.L. Chu, C.H. Lee, and G.A. Gruber, "The Preparation and Properties of Amorphous Silicon Nitride Films," J. Electrochem. Soc.: Solid State Science, Vol. 114, No. 7, pp. 717-722, 1967.

8. T. A. Shankoff and E. A. Chandross, "High Resolution Tungsten Patterning Using Buffered, Mildly Basic Etching Solutions," Journal Electrochemical Society: Solid-State Science and Technology, Vol. 122, No. 2, pp. 294-298, Feb. 1975.

9. E.G.Zubler and F.A. Mosby, "An Incandescent Lamp with Virtually 100\% Lumen Maintenance," Illum. Eng. Soc., Vol. 54, p. 734, 1959. 